Supplementary Information for

\title{
Narrowing working voltage window to improve layered GeP anode cycling performance for lithium-ion batteries
}

Hailin Shen ${ }^{\mathrm{a} \#}$, Yu Huanga", Yukai Chang ${ }^{\mathrm{b} \sharp}$, Ronghui $\mathrm{Hao}^{\mathrm{a}}$, Zhongtao $\mathrm{Ma}^{\mathrm{a}}$, Kai Wua Pengshan $\mathrm{Du}^{\mathrm{a}}$, Bingkun Guo ${ }^{\mathrm{a}}$, Yingchun Lyu ${ }^{\mathrm{a}}$, Peng Wang, , Hangsheng Yang, Qianqian $\mathrm{Li}^{\mathrm{a}^{*}}$, Hongtao Wang ${ }^{\mathrm{c}}$, Zhongyuan Liu ${ }^{\mathrm{b}}$, Anmin $\mathrm{Nie}^{\mathrm{a}, \mathrm{b}}{ }^{*}$

${ }^{a}$ Materials Genome Institute, Shanghai University, Shanghai, China;

${ }^{b}$ Center for High Pressure Science, State Key Laboratory of Metastable Materials Science and Technology, Yanshan University, Qinhuangdao 066004, China

${ }^{c}$ Center for X-mechanics, Zhejiang University, Hangzhou 310027, China

${ }^{d}$ State Key Laboratory of Silicon Materials, School of Materials Science and Engineering, Zhejiang University, Hangzhou 310027, China

\#These authors contributed equally to the work.

To whom correspondence should be addressed. E-mails: anmin@ysu.edu.cn (AnminNie), htw@zju.edu.cn (Hongtao Wang), and qianqianli@shu.edu.cn (Qianqian Li) 

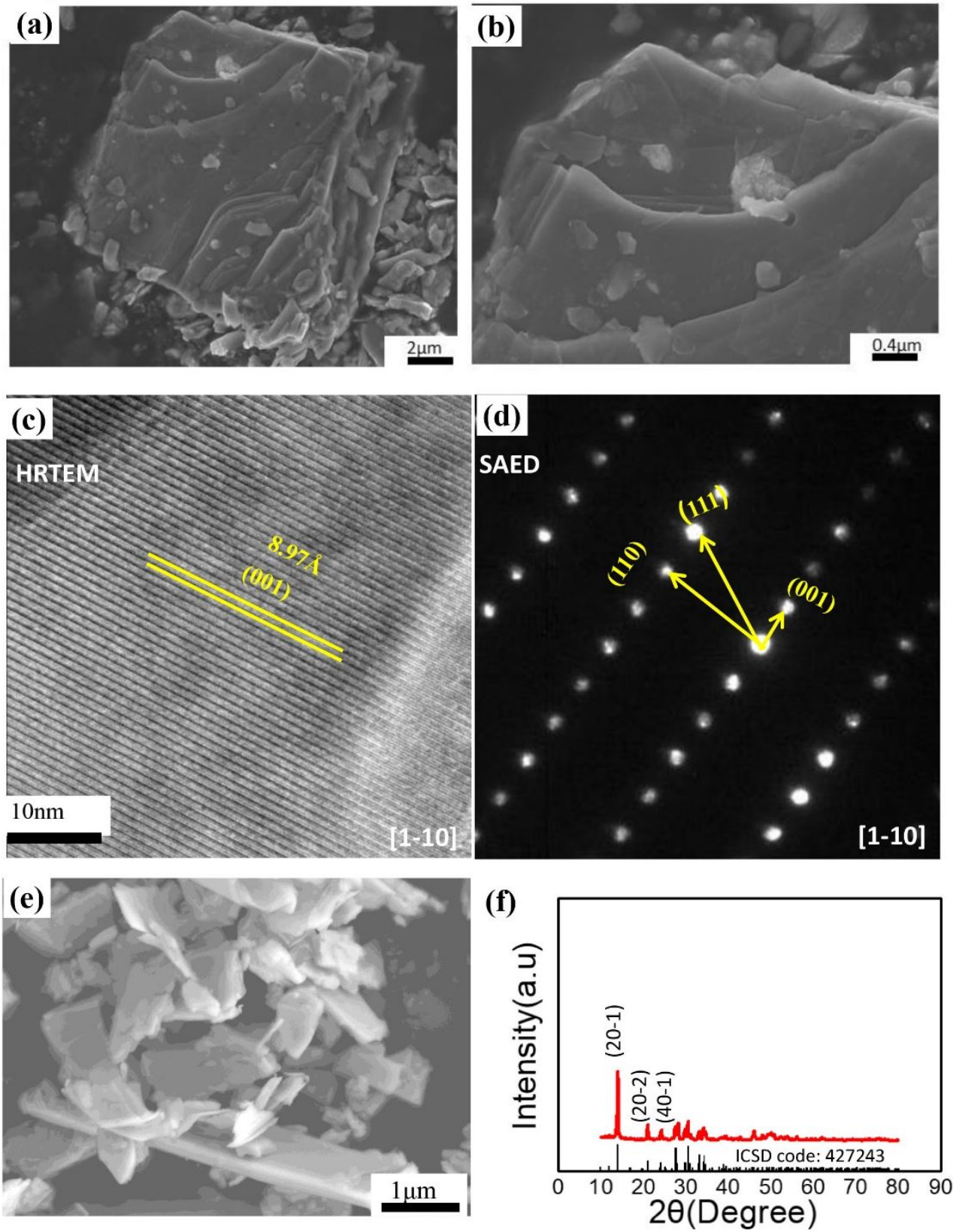

Figure S1. Characterization of GeP flakes. (a-b) SEM images of the bulk GeP. (c) Typical high-resolution transmission electron microscopy (HRTEM) images of individual GeP nanoflake. (d) The selected area election diffraction (SAED) pattern of GeP viewed along [1-10] direction. (e) SEM image of GeP nanoflakes. (f) XRD pattern of obtained GeP powders, which matched well by the theoretical crystal structure with number of ICSD code:427243. 


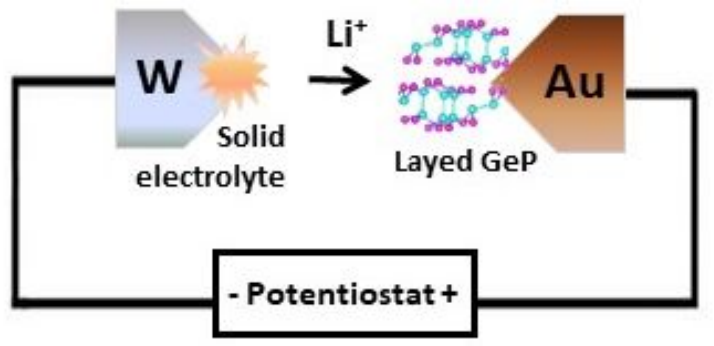

Figure S2. Schematic nanobattery setup used in the in-situ TEM. 
Table S1

Comparison of the initial coulombic efficiency of different electrodes for LIBs

\begin{tabular}{ccc}
\hline $\begin{array}{c}\text { Anode } \\
\text { material }\end{array}$ & $\begin{array}{c}\text { Initial } \\
\text { Coulombic efficiency(\%) }\end{array}$ & Reference \\
\hline $\mathrm{GeP}_{3} / \mathrm{C}$ & 73.8 & {$[1]$} \\
$\mathrm{SiP}$ & 70 & {$[2]$} \\
$\mathrm{MoP}$ & 69 & {$[3]$} \\
$\mathrm{GeP}_{3}$ & 78.5 & {$[4]$} \\
$\mathrm{GeP}$ & 89 & This work \\
$\mathrm{Commercial}$ & 85 & {$[5]$} \\
$\mathrm{graphite}$ & & \\
$\mathrm{Ge} / \mathrm{C}$ & 65.5 & {$[6]$} \\
$\mathrm{P} / \mathrm{C}$ & 77.46 & {$[7]$} \\
$\mathrm{Si} / \mathrm{graphite}$ & 58 & {$[8]$} \\
$\mathrm{SnP}$ & 78.9 & {$[9]$} \\
\hline
\end{tabular}




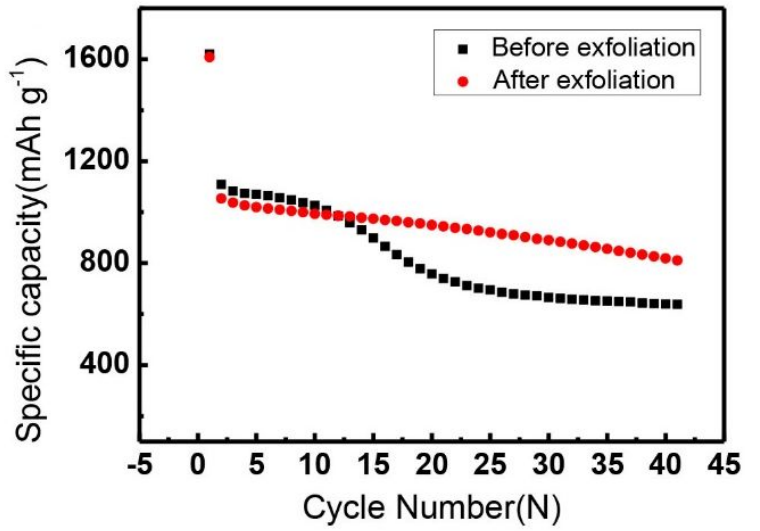

Figure S3. Cycle performances of GeP anodes with the cutoff voltage of 0.001 $2 \mathrm{~V}$ before and after stripping at $0.1 \mathrm{~A} \mathrm{~g}^{-1}$. 

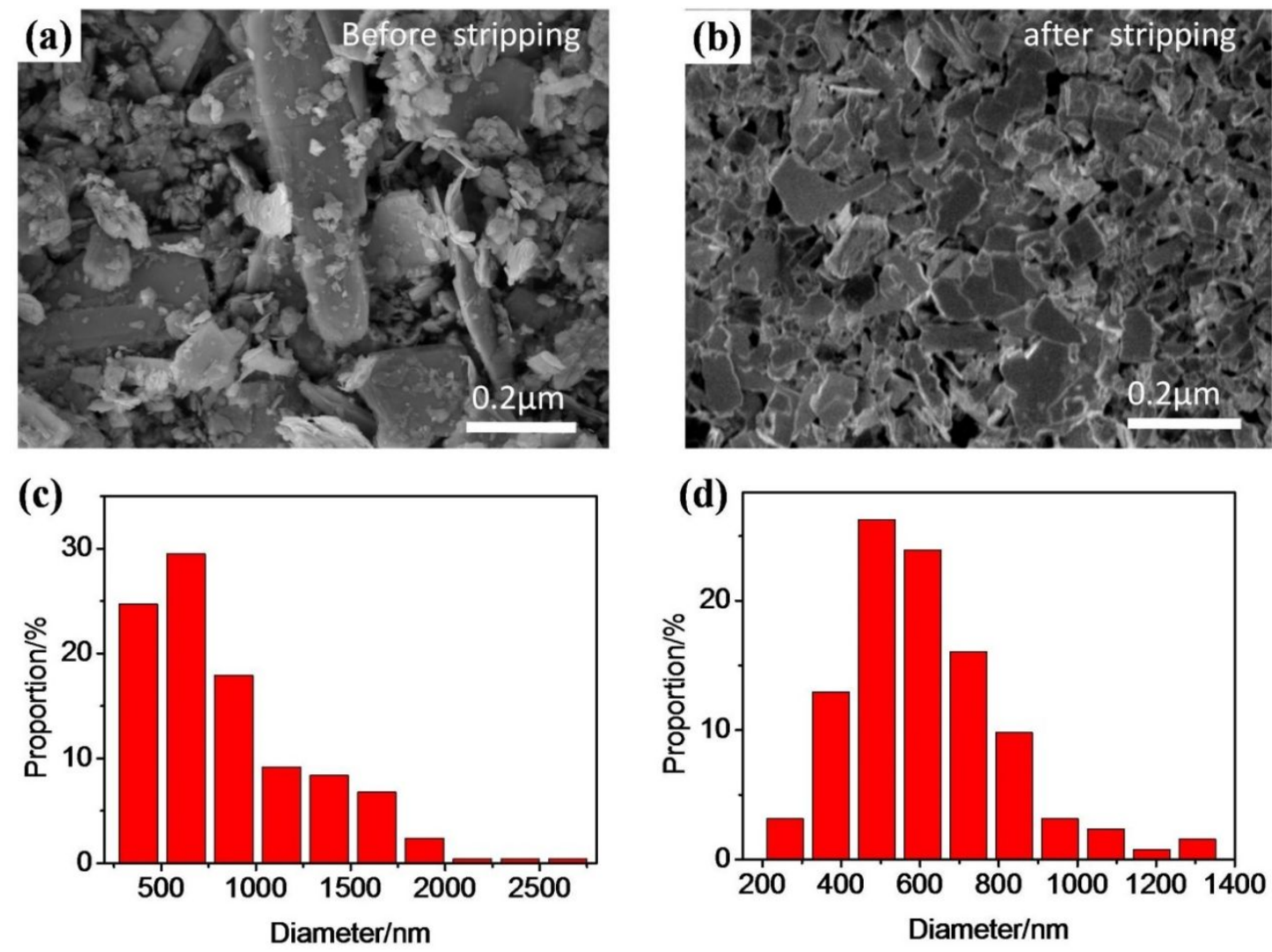

Figure S4. SEM images of GeP nanoflakes (a) before stripping, (b) after stripping. The size distribution of GeP nanoflakes (c) before stripping, (d) after stripping. 

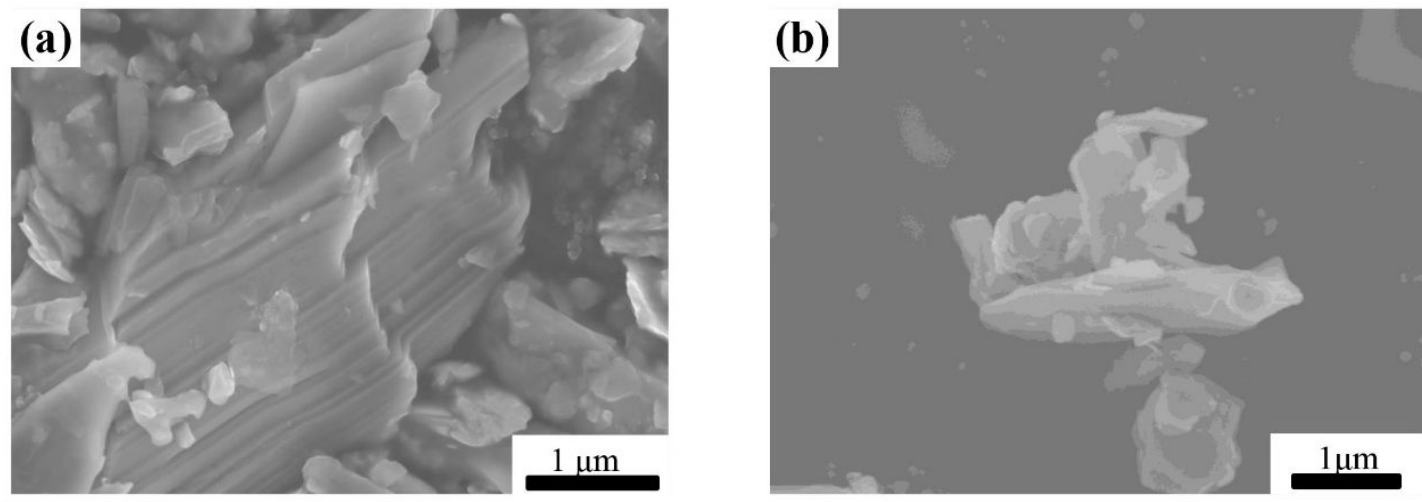

Figure S5. The cross-section images of GeP nanoflakes (a) before stripping, (b) after stripping. 
(a)

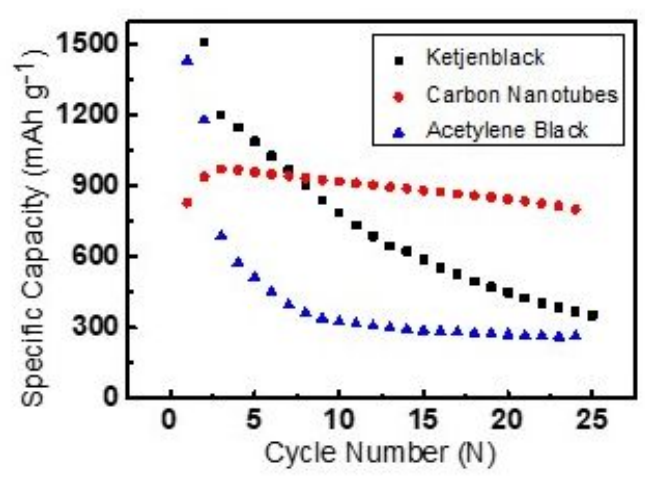

(b)

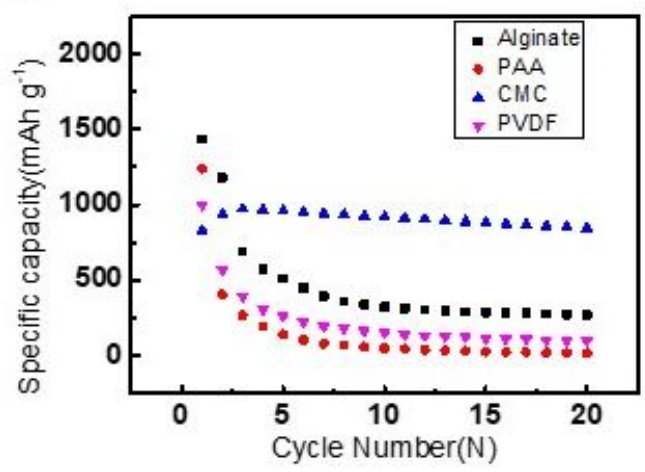

Figure S6. (a) Cycle performances of $\mathrm{GeP}$ anodes with different conductive agents at $0.4 \mathrm{~A} \mathrm{~g}^{-1}$. (b) Cycle performances of GeP anodes with different binders at $0.4 \mathrm{~A} \mathrm{~g}^{-1}$ 

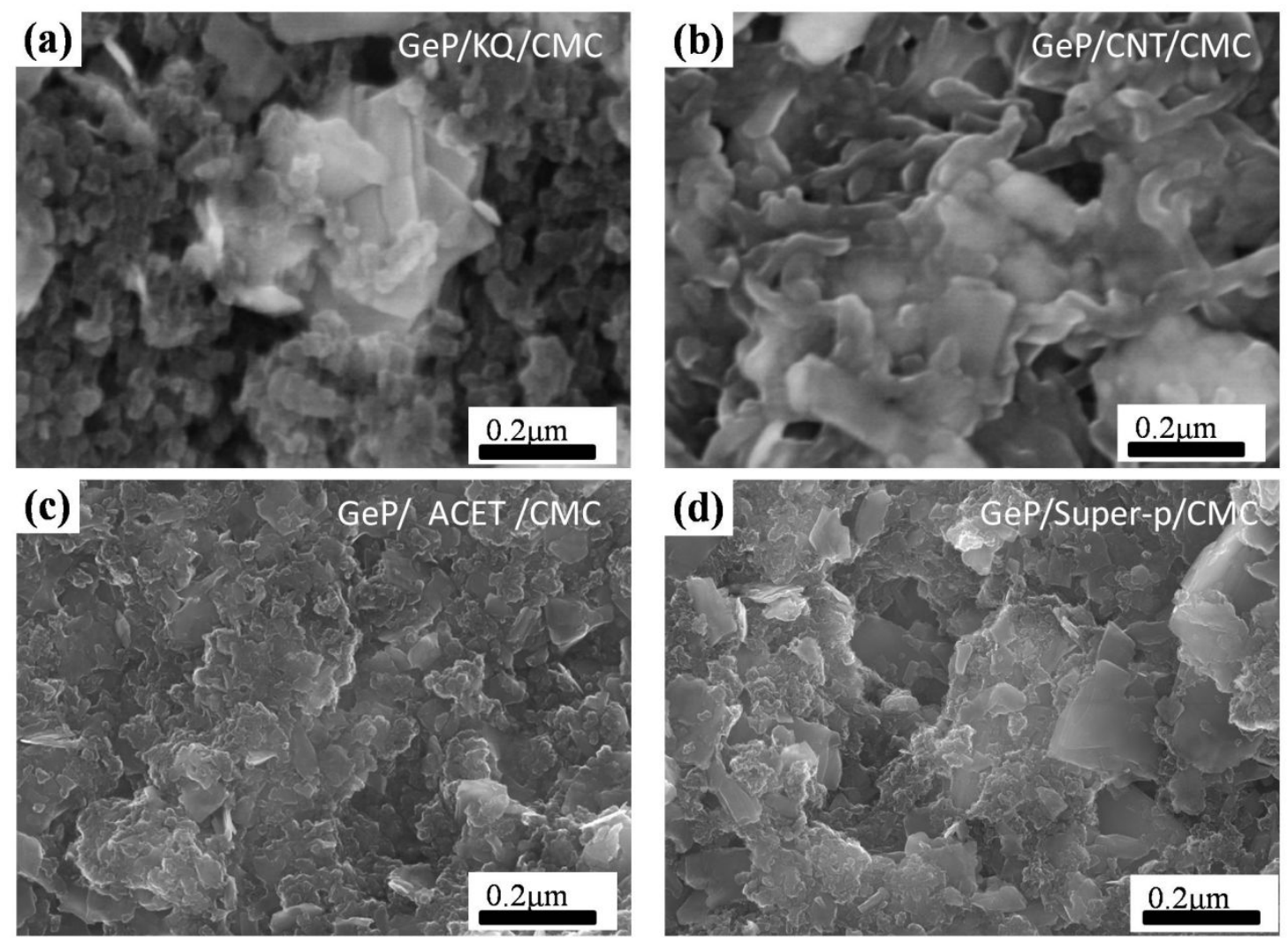

Figure S7. SEM images of GeP with different conductive agents, (a) Ketjenblack,

(b) Carbon nanotube, (c) Acetyleneblack, (d) Super-p. 

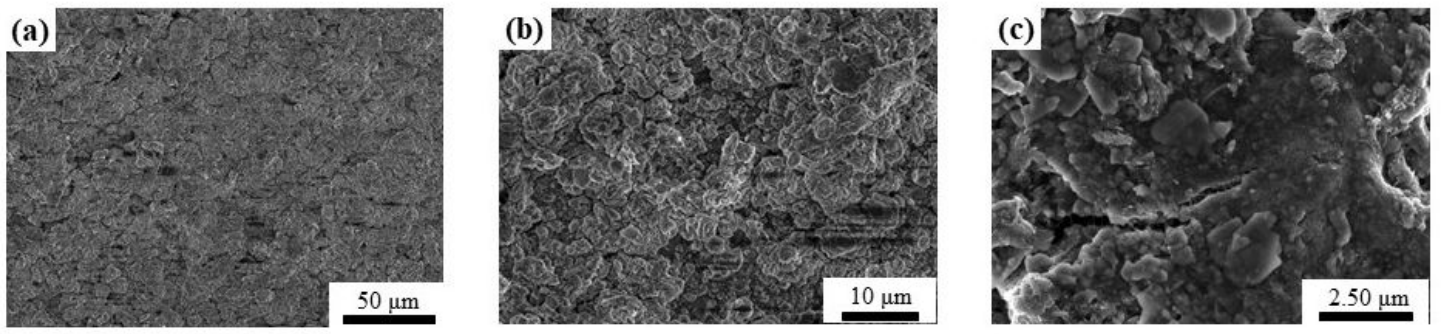

Figure S8. (a-c) SEM of the GeP/CNT/CMC electrode surface morphology in lithiation/delithiation cycles after 100 cycles at various magnification within the potential window of $0.001-3 \mathrm{~V}$. 


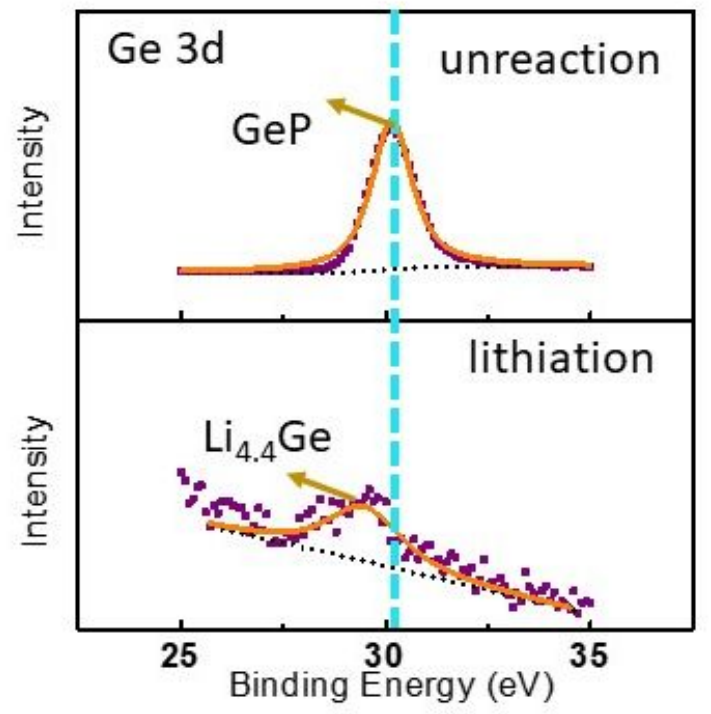

Figure. S9. XPS for the GeP/CNT/CMC electrode before and after lithiation for Ge 3d. 


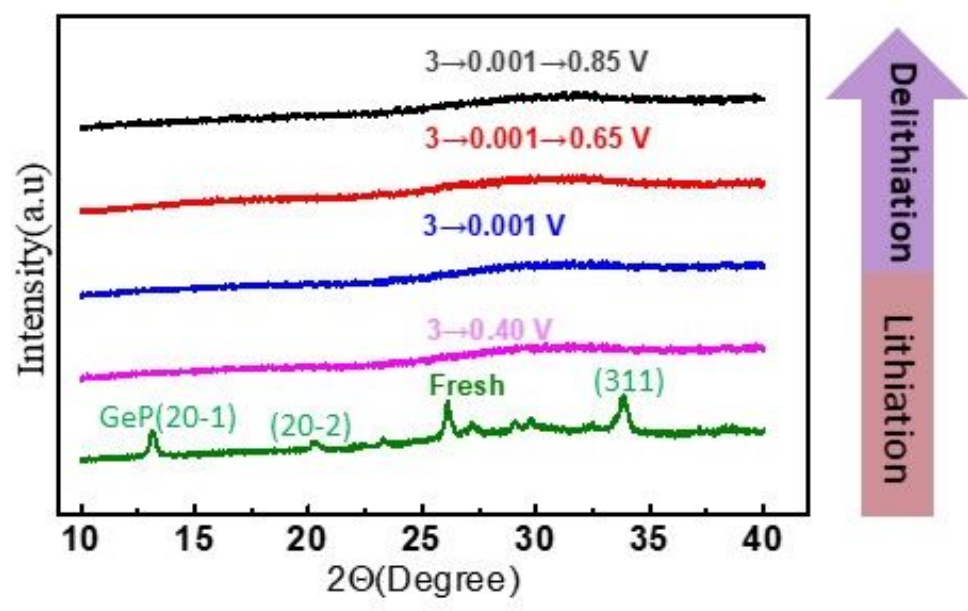

Figure S10. The XRD patterns of the GeP/CNT/CMC electrodes taken from different reaction stages in first discharge/charge cycle. 


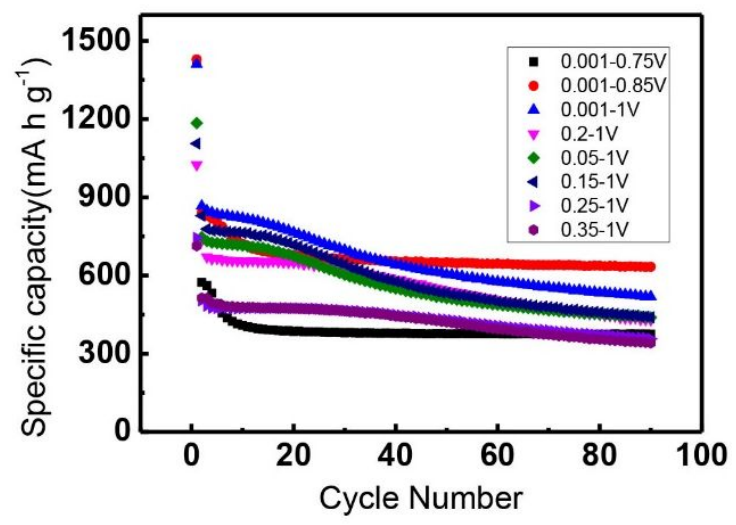

Figure S11. Electrochemical performance comparisons among series of working potential of $0.001-0.75 \mathrm{~V}, 0.001-0.85 \mathrm{~V}, 0.001-1 \mathrm{~V}, 0.05-1 \mathrm{~V}, 0.15-1 \mathrm{~V}, 0.25-1$ $\mathrm{V}, 0.35-1 \mathrm{~V}$ at $0.1 \mathrm{~A} \mathrm{~g}^{-1}$. 

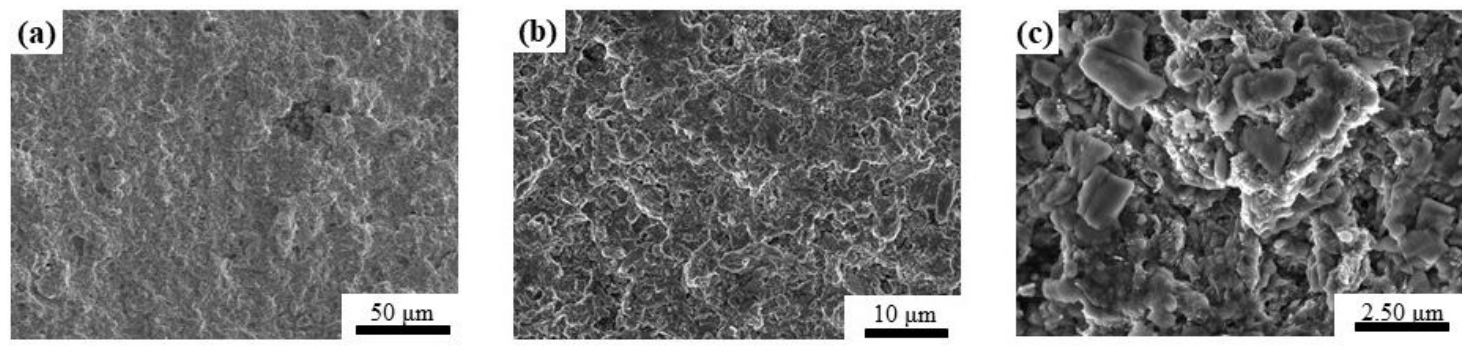

Figure S12. (a-c) SEM of the GeP/CNT/CMC electrode surface morphology in lithiation/delithiation cycles after 100 cycles at various magnification within the potential window of $0.001-0.85 \mathrm{~V}$. 


\section{Table S2}

Fitted equivalent- circuit resistance data for GeP/CNT/CMC after 100 charge-discharge cycles between the potential window of $0.001-0.85 \mathrm{~V}$ and $0.001-3 \mathrm{~V}$.

\begin{tabular}{lccc}
\hline condition & $\mathrm{R}_{\mathrm{S}}(\Omega)$ & $\mathrm{R}_{\mathrm{SE}}(\Omega)$ & $\mathrm{R}_{\mathrm{ct}}(\Omega)$ \\
\hline $0.001-0.85 \mathrm{~V}$ & 3.531 & 11.23 & 58.85 \\
\hline $0.001-3 \mathrm{~V}$ & 0.815 & 23.45 & 94.9 \\
\hline
\end{tabular}




\section{Reference}

(1) Qi, W.; Zhao, H.; Wu, Y.; Zeng, H.; Tao, T.; Chen, C.; Kuang, C.; Zhou, S.; Huang, Y. Facile Synthesis of Layer Structured $\mathrm{GeP}_{3} / \mathrm{C}$ with Stable Chemical Bonding for Enhanced Lithium-Ion Storage. Sci Rep 2017, 7, 43582.

(2) Reinhold, R.; Mikhailova, D.; Gemming, T.; Missyul, A. B.; Nowka, C.; Kaskel, S.; Giebeler, L. Silicon Monophosphide as a Possible Lithium Battery Anode Material. J Mater Chem A 2018, 6 (41), 19974-19978.

(3) Wang, X.; Sun, P.; Qin, J.; Wang, J.; Xiao, Y.; Cao, M. A Three-Dimensional Porous MoP@ C Hybrid as a High-Capacity, Long-Cycle Life Anode Material for Lithium-Ion Batteries. Nanoscale 2016, 8 (19), 10330-10338.

(4) Nam, K.-H.; Jeon, K.-J.; Park, C.-M. Layered Germanium Phosphide-Based Anodes for High-Performance Lithium- and Sodium-Ion Batteries. Energy Storage Maters 2019, 17, 78-87.

(5) Li, W.; Li, H.; Lu, Z.; Gan, L.; Ke, L.; Zhai, T.; Zhou, H. Layered Phosphorus-Like $\mathrm{GeP}_{5}$ : A Promising Anode Candidate with High Initial Coulombic Efficiency and Large Capacity for Lithium Ion Batteries. Energ Environ Sci 2015, 8 (12), 3629-3636.

(6) Ngo, D. T.; Kalubarme, R. S.; Le, H. T. T.; Fisher, J. G.; Park, C.-N.; Kim, I.-D.; Park, C.-J. Carbon-Interconnected Ge Nanocrystals as an Anode with Ultra-Long-Term Cyclability for Lithium Ion Batteries. Adv Funct Mater 2014, 24 (33), 5291-5298.

(7) Marino, C.; Debenedetti, A.; Fraisse, B.; Favier, F.; Monconduit, L. ActivatedPhosphorus as New Electrode Material for Li-Ion Batteries. Electrochem Commun 2011, 13 (4), 346-349. 
(8) Zhou, X.; Yin, Y. X.; Wan, L. J.; Guo, Y. G. Self-Assembled Nanocomposite of Silicon Nanoparticles Encapsulated in Graphene through Electrostatic Attraction for Lithium-Ion Batteries. Adv Energy Mater 2012, 2 (9), 1086-1090.

(9) Park, J.-W.; Park, C.-M. Electrochemical Li Topotactic Reaction in Layered $\mathrm{SnP}_{3}$ for Superior Li-Ion Batteries. Sci Rep 2016, 6, 35980. 